\title{
Young adults' perceptions of breastfeeding in public: a focus group study
}

Ka-Huen Yip ${ }^{a, *}$, Yuk-Chiu Yip ${ }^{a}$, Catherine Man-Yin Leea, Mei-Yi Siuª, Shu-Ying Ngaia, Mei-Kuen Chow

${ }^{a}$ School of Health Sciences, Caritas Institute of Higher Education, Hong Kong, China

${ }^{b}$ School of Nursing, The University of Hong Kong, Hong Kong, China

Received: 12 September 2019; Accepted: 22 November 2019; Published: 20 September 2020

Abstract: Objective: To elucidate the perceptions of young Chinese adults aged 18-25 toward adopting breastfeeding in public in Hong Kong Special Administrative Region (Hong Kong), China.

Methods: A descriptive and exploratory study design was adopted. Participants were recruited by convenience sampling. Focus group data were collected from three semi-structured interviews. Responses from 18 participants were audiotaped, transcribed verbatim, coded, and analyzed using content analysis.

Results: Four themes emerged that described the young adults' perceptions and barriers toward breastfeeding practices: (1) adaptability of self and others, (2) cultural deem and practice, (3) convenience and connection, and (4) education and advocacy. Interestingly, all respondents (male and female) are aware of the benefits of breastfeeding. Their negative perceptions toward breastfeeding initiation and continuation originate from factors that are essentially extraneous to the mother-infant dyads (such as the breadth and intensity of sociopolitical support, the dominant social beliefs and norms, and the inadequacy of breastfeeding education in adolescent years).

Conclusions: In general, the young adult's perception of breastfeeding is dominated by sociopolitical and cultural-related factors. In particular, sexualization of breasts as a deep-rooted belief in the Chinese context negatively affects young adults' perceptions and limits their breastfeeding practices.

Keywords: breastfeeding $\bullet$ young adult $\bullet$ perceptions $・$ Chinese $\bullet$ Hong Kong

(c) Shanxi Medical Periodical Press.

\section{Introduction}

The benefits of breastfeeding for mothers and infants have been extensively studied and recognized worldwide. ${ }^{1,2}$ The breastfeeding initiation rates in most developed countries (such as Australia, Canada, Denmark,
Norway, and Sweden) have reached a prevalence of about $90 \% .^{3-8}$ However, the rate in Hong Kong Special Administrative Region (Hong Kong), China has stopped improving. Substantial improvement has been observed over the past 30 years since 1981, when fewer than $20 \%$ of mothers breastfed their infants, as about $80 \%$

How to cite this article: Yip KH, Yip YC, Lee CMY, Siu MY, Ngai SY, Chow MK. Young adults' perceptions of breastfeeding in public: a focus group study. Front Nurs. 2020;3:217-226. 
of mothers currently initiate breastfeeding. ${ }^{9-11}$ About $20 \%$ of those mothers reported exclusive breastfeeding during the infant's first 3 months. ${ }^{12}$ Nursing researchers are concerned about the lack of recent progress plus the recent high breastfeeding attrition rate in light of the Hong Kong Special Administrative Region's (HKSAR) public rhetoric and efforts to promote breastfeeding. ${ }^{12-14}$ Findings from a recent prospective cohort study indicated that premature weaning is ubiquitous in Hong Kong, and about one-half of the mothers who start breastfeeding discontinue it within the first 3 months. ${ }^{15}$ This surprisingly short duration and the low rate of exclusive breastfeeding clarify that Hong Kong is far from the international breastfeeding recommendations. ${ }^{16-18}$ To understand the reasons underlying Hong Kong's breastfeeding initiation rate and early weaning, exploratory and contextual studies are needed that focus on the barriers to breastfeeding that mothers are facing. ${ }^{2}$ This article examined young adults' perceptions of breastfeeding. There is substantial evidence that young adults are at risk of choosing early weaning. The age distribution of breastfeeding initiators in Hong Kong is about four times lower among mothers aged 18-24 years in comparison with older mothers. ${ }^{11}$ Another study highlights that relatively young, primipara, or mothers who have never breastfed are highly likely to choose premature weaning. ${ }^{12}$ Furthermore, a population-based survey found that mothers aged 18-29 had the least amount of knowledge about the international breastfeeding recommendations. ${ }^{19}$ Almost $50 \%$ of mothers younger than 26 years had never breastfed, and twice as many mothers aged 26 or older reported that they had breastfed longer than 6 weeks. ${ }^{20}$ In sum, previous research shows the age differences in breastfeeding behaviors and patterns. One possible reason for the differences might be variation in some age-related sociocultural and/or psychosocial factors that influence women's perceptions of and attitudes toward breastfeeding.

To explore the reasons behind Hong Kong's breastfeeding patterns among young mothers, an electronic literature search was performed, which focused on evidence obtained from primary studies on young adults' (aged 18-25) perceptions of and attitudes toward breastfeeding. Most of these studies were set in the West. ${ }^{21-29}$ However, perceptions of breastfeeding have been found to vary by sociocultural background, ${ }^{20,30-32}$ and their generalizability to Chinese cultures in Asia is not clear. The Western studies tended to focus on older adults (about 26-41 of age), ${ }^{22-24,29}$ and the Chinese mainland and Hong Kong studies often focused on small numbers of young adults (about 6-14\%). ${ }^{11,33-35}$ Because previous relevant studies are scarce, and this article took an exploratory and narrative approach to learn about the breastfeeding perceptions of young adults, who, because of their relatively high risk of early weaning, should be targeted for customized breastfeeding interventions. To ensure the validity of the findings, and because fathers' opinions and support have been found to influence the mothers' decisions to initiate and continue breastfeeding, this article involved male and female young adults. ${ }^{36-38}$ For young mothers, breastfeeding is more than a simple decision about their infants' nutritional needs. ${ }^{39}$ The decision-making process begins with the initial intention and continues through the initiation phase to the choice of continuing breastfeeding. The predictors, particularly the facilitators and constraints, have not yet been thoroughly investigated. ${ }^{40}$ Several conceptual frameworks have been widely applied to understanding and analyzing breastfeeding patterns, such as the theory of reasoned action, theory of planned behavior, and the breastfeeding self-efficacy theory. The perceptions of young adults, including their attitudes, subjective norms, and perceptions of the relationship between the mother-infant dyad and community/societal environment, which are crucial to health behaviors, are at the core of these frameworks. ${ }^{41,42}$ Consequently, by investigating perceptions, this article might reveal salient barriers of psychosocial and/or sociocultural origin that are unique to the target population. Whether and/or how breastfeeding might be perceived as inconvenient (vs. convenient), embarrassing (vs. unabashed), inappropriate in certain situations (vs. accepted), and restrictive of mothers' freedom (vs. unconfined) remain to be elucidated. In addition, Hong Kong is a unique sociocultural context in which traditional Chinese culture functions within a highly Westernized approach to development. Young mothers' breastfeeding intentions might be strongly influenced by their elder family members' opinions within families that adhere to Confucian principles of respect and love..$^{34,43}$ However, the majority of young mothers continue fulltime employment after their babies are born in an intense work culture that highly values productivity and financial gain. ${ }^{44}$ Mandatory maternity leave is relatively short (currently it is a maximum of 10 weeks), and the need to return to work might influence young mothers' attitudes toward continuing breastfeeding. ${ }^{45}$ Thus, this article might yield rich qualitative knowledge on young adults' perceived facilitators and constraints regarding breastfeeding through their first-hand and accounts. This article's exploratory and descriptive qualitative approach might inform two ways to optimize current breastfeeding promotional efforts. ${ }^{46,47}$ First, nurse midwives might use the information about facilitators and constraints regarding breastfeeding initiation and reason for early weaning specifically for their young adult parents. Breastfeeding interventions might be customized to respond to the modifiable reasons for not initiating..$^{48}$ 
Second, this article might help nurses to strengthen their strategies for promoting autonomous self-determined breastfeeding. Finding ways to increase mothers' level of interest in and enthusiasm for the practice would be useful because mothers with a genuine interest in it are relatively more committed to the practice and more satisfied with better psychological well-being. . $^{36,49-51}$

\section{Methods}

\subsection{Objective}

This article aims to explore the perceptions of breastfeeding among young adults in breastfeeding: their knowledge, attitudes, and exposure toward breastfeeding among young adults in Hong Kong.

Participants were recruited in this article using the convenience sampling method. There were three recruitment criteria for eligible participants: (a) Hong Kong residents and recruited in Hong Kong; (b) aged between 18 and 25 years old; (c) able to understand and speak in Cantonese.

\subsection{Data collection}

Focus group discussions were held in November and December 2018. We developed a discussion guide (Table 1) based on a literature review on perception and practice of breastfeeding in public, and related physical and psychosocial health perceptions. ${ }^{52-57}$

There were topics in the guide such as perceptions of breastfeeding (e.g., annoying and disgusting or a normal way of feeding a baby in public and breastfeeding in public as acceptable). The interviews of the participants were arranged in a private environment in which there were no other people except the interviewees and the focus group members. The room for holding the focus group meeting was a private air-conditioned one within the Caritas Institute of Higher Education and without disturbance. The interviews were conducted through face-to-face and semi-structured group meetings with the participants.

The first author $(\mathrm{KH})$, who had received training in conducting and analyzing focus group discussions, moderated each semi-structured focus group discussion. The focus group meetings were facilitated by the first author, 1 male and 1 female research assistants (RAs). The first author started the focus group meeting by explaining the purpose of the meeting to the group members and the interviewees and using the discussion guide to elicit the discussion in the group. All of the interviewees participated and conveyed their own perception in the discussion. The RAs in the whole discussion sessions took reflective handwritten notes of verbal and nonverbal responses and recorded further elaboration on points made. Every attempt was made to achieve data saturation by guiding semi-structured discussions within each focus group. Participants completed a short questionnaire on demographic information, with written consent. The length of the discussion was about 60-80 min.

\subsection{Ethical consideration}

The Research and Ethics Committee, Caritas Institute of Higher Education approved this study prior to commencement. A total of 18 participants were recruited and agreed to take part in the study. They were informed that if they felt uncomfortable during the interviews, they were allowed to leave the study at any time. To protect the interviewees' privacy, the study used anonymous identifications, and all audiotaped data were secured.

\subsection{Data analysis and rigor}

We audiotaped and transcribed verbatim all focus group discussions and analyzed them using a thematic approach, ${ }^{58}$ aided by the NVivo 12 qualitative software..$^{59}$ The second author (YC), third author (CMY), and fifth author (SY) reviewed all transcripts and derived a set of themes and subthemes from the discussions, and the

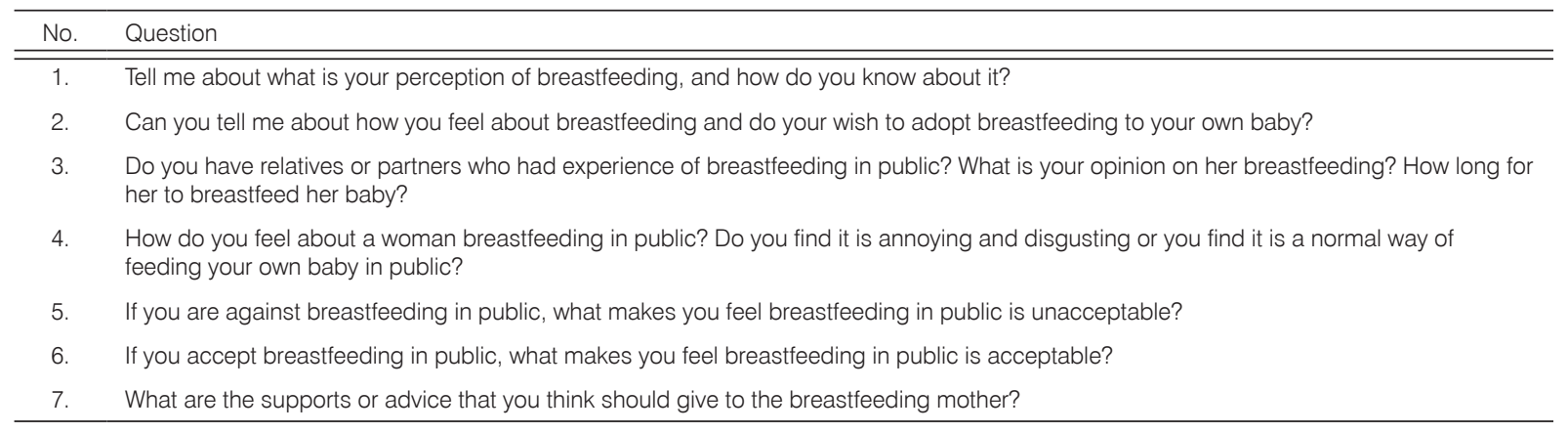

Table 1. Focus group question guide. 
fourth and sixth authors (MY and MK) independently reviewed all transcripts and the appropriateness of the themes and subthemes derived by the first author. Disagreements on themes and subthemes were resolved through discussions. The second, third, and fifth authors then coded all the transcripts by categorizing relevant statements in the transcripts under themes and subthemes. ${ }^{60}$ The first author reviewed the coded statements. Disagreements on the codes were resolved through discussions. Two other authors (MY and MK) verified the results of the analysis after reading through all transcripts. We also examined the findings in terms of the type of school from which the participant received her/his secondary education, and any relatives or partners who had experience of breastfeeding in public.

\section{Results}

\subsection{Demographic characteristics}

We conducted 3 focus group discussions involving a total of 18 participants (9 males and 9 females), in 3 groups with 6 participants per group. Participants were between 20 and 25 years old (Table 2). Most of the participants were employed $(50 \%), 1$ was unemployed, and the others were students (44\%). One had acquired secondary education, 8 had post-secondary education, 5 were graduates with a bachelor's degree, and 4 had post-graduate education (Table 2). About 33\% (6) of the participants reported their monthly income was US\$1,250-\$2,549, 5 earned lower than US\$1,249, 3 were earned more than US\$2,500, and others had no monthly income (Table 2). The perception toward public breastfeeding among young adults was categorized into four main themes: (a) adaptability of self and others, (b) cultural deem and practice, (c) convenience and connection, and (d) educational and advocacy.

\subsubsection{Adaptability of self and others}

An impact arising from the data was that all the participants typically described the benefit of breastfeeding to both mothers and their own babies. All female participants were willing to breastfeed their babies in the future, for it is the mothers' natural instinct to provide nutrients for their babies. The attraction to breastfeed their babies in the future was that a close relationship could be built up between the mothers and the babies through intimate bodily contact. One university female student stated:

"If I were a mother, I would breastfeed my baby, as it is best way to provide nutrition for babies." (P12: female, age 24)
Some participants had a similar response: "This is the mother's own choice ... I think it (breastfeeding) is even if the mother expose her breast." (P15: female, age 21)

The attitude and perceptions of all male participants expressed they would support breastfeeding if their partners' health condition were suitable. A man who is a post-secondary student mentioned:

"I will definitely support if her physical condition allowed for it.” (P16: male, age 23)

\begin{tabular}{|c|c|c|c|c|c|c|}
\hline \multirow[t]{2}{*}{ Characteristic } & \multicolumn{2}{|c|}{ All $(n=18)$} & \multicolumn{2}{|c|}{ Male $(n=9)$} & \multicolumn{2}{|c|}{ Female $(n=9)$} \\
\hline & $n$ & $\%$ & $n$ & $\%$ & $n$ & $\%$ \\
\hline \multicolumn{7}{|l|}{ Occupation } \\
\hline Student & 8 & 44 & 3 & 34 & 5 & 55 \\
\hline Employed & 9 & 50 & 6 & 66 & 3 & 34 \\
\hline Unemployed & 1 & 6 & 0 & 0 & 1 & 11 \\
\hline \multicolumn{7}{|l|}{ Educational level } \\
\hline Secondary & 1 & 6 & 1 & 12 & 0 & 0 \\
\hline Post-secondary & 8 & 44 & 5 & 55 & 1 & 12 \\
\hline $\begin{array}{l}\text { Bachelor's } \\
\text { degree }\end{array}$ & 5 & 28 & 0 & 0 & 5 & 55 \\
\hline Post-graduate & 4 & 22 & 3 & 33 & 3 & 33 \\
\hline \multicolumn{7}{|l|}{ Age } \\
\hline 18 & 0 & 0 & 0 & 0 & 0 & 0 \\
\hline 19 & 0 & 0 & 0 & 0 & 0 & 0 \\
\hline 20 & 2 & 11 & 1 & 11 & 1 & 11 \\
\hline 21 & 2 & 11 & 1 & 11 & 1 & 11 \\
\hline 22 & 3 & 17 & 0 & 0 & 3 & 34 \\
\hline 23 & 3 & 17 & 2 & 22 & 1 & 11 \\
\hline 24 & 3 & 17 & 2 & 22 & 1 & 11 \\
\hline 25 & 5 & 27 & 3 & 34 & 2 & 22 \\
\hline \multicolumn{7}{|l|}{ Marital status } \\
\hline Single & 18 & 100 & 9 & 100 & 9 & 100 \\
\hline Married & 0 & 0 & 0 & 0 & 0 & 0 \\
\hline Divorced & 0 & 0 & 0 & 0 & 0 & 0 \\
\hline Widowed & 0 & 0 & 0 & 0 & 0 & 0 \\
\hline \multicolumn{7}{|c|}{ Monthly income, US\$ } \\
\hline 0 & 4 & 22 & 1 & 12.5 & 3 & 30 \\
\hline$<1,249$ & 5 & 28 & 2 & 25 & 3 & 30 \\
\hline $1,250-2,549$ & 6 & 33 & 4 & 50 & 2 & 20 \\
\hline$>2,500$ & 3 & 17 & 1 & 12.5 & 2 & 20 \\
\hline \multicolumn{7}{|l|}{ Religious belief } \\
\hline Nil & 17 & 94 & 8 & 89 & 9 & 100 \\
\hline Christian & 1 & 6 & 1 & 11 & 0 & 0 \\
\hline Catholic & 0 & 0 & 0 & 0 & 0 & 0 \\
\hline Buddhism & 0 & 0 & 0 & 0 & 0 & 0 \\
\hline
\end{tabular}

Table 2. Characteristics of focus groups discussion. 
Although another man had a similar view to him, he emphasized that he did not agree to his partner breastfeeding in public without privacy: "I support breastfeeding, and support my partner to breastfeed, but I prefer no breastfeed in public if there is no nursery room." (P01: male, age 24)

Young adults support breastfeeding. Both male and female participants accept breastfeeding in public only under specific conditions, like having privacy. All female participants were willing to try breastfeeding for their babies in the future if their health conditions are suitable. The partners' health condition was a key factor to support breastfeeding among the male gender in Hong Kong.

\subsubsection{Cultural consideration and practice}

The culture, both traditional Chinese and local of young adults, was another factor that affects their thoughts and behaviors. All participants agreed that traditional Chinese culture (behaviors of citizens) would significantly affect the mother's intention of breastfeeding. Although everyone accepted breastfeeding in public if the mothers would cover up their breasts, eight participants struggled with similar thoughts about social norms and traditional Chinese culture:

\footnotetext{
"The social norm does not allow her to breastfeed in public." (P14: female, age 22)

"In terms of culture, Chinese have a conservative mind set; for example, females are told to dress in a conservative way." (P16)
}

Some female participants would not engage in breastfeeding in public although they support breastfeeding. "I can't accept myself to breastfeed in public, but it is okay if others do so." (P05: female, age 22)

"Female has an embarrassed feeling on breastfeeding, so she is not willing to breastfeed in public area." (P15)

The majority of the participants noted that the local Hong Kong culture focused on having a speedy pace of life, and emphasized having "speed-up-tosave-some-time" lifestyles. They chose bottle feeding with formula milk instead of breastfeeding as they considered bottle feeding more convenient and less time-consuming, especially in the Chinese culture. P16 stated,

"Breastfeeding takes time, but feeding a baby with formula milk is less time consuming, and it is also convenient."
Two female participants agreed with P04's comment that breast pertained a linkage to the concept of sex:

"International sexualized female breasts always link up female's breasts and sex." (P04: female, age 23)

Some young adults prefer artificial feeding rather than breastfeeding based on their own personal lifestyle. Some people in Hong Kong do not accept breastfeeding in public due to their apparent misperception of ignoring the breasts for providing milk for babies. There is a tendency to associate breasts and sex due to the norms of traditional Chinese culture. Sexualisation results in low acceptance of breastfeeding, as breasts should not be exposed, and it is also regarded as disrespectful behavior to others. The norms of society affect the decisions of a mother about whether or not to breastfeed in public.

\subsubsection{Convenience and connection}

Numerous participants reported that convenient facility was another factor that would affect mothers' decisions to breastfeed, including the provision of nursery rooms in public areas like shopping malls or the workplace environment. The data on different types of public facilities provided by the government revealed that all participants mentioned there were few or no nursery rooms. Similarly, the observation was the same for some shopping malls. For those with nursery rooms, the rooms were difficult to locate, and spaces were inadequate to conduct breastfeeding.

"There are not enough facilities for breastfeeding. Even in big shopping malls, nursery rooms are located in hidden place and are small." (P13: male, age 21)

The provision of nursery rooms in the workplace environment would be an incentive for working mothers to conduct breastfeeding, such as to provide a fridge for breast milk storage. Three female participants recognized that breastfeeding would be more accessible if more facilities could be provided in the workplace:

"I will be more willing to continue breastfeeding in the future if there is a room provided for me in my workplace, since I can feel as though my company is supporting me to do this." (P07: female, age 25)

"Allow more break time for expressing breast milk." (P04)

"I will have my privacy." (P09: female, age 20)

Further, connecting the young adults to an understanding and practice of breastfeeding is equally 
important. The media, with their broadcasting to a vast coverage of all walks of life, can play a vital role in promoting breastfeeding in the society. All participants mentioned that there was a limited promotion of breastfeeding through either mass or social media. "There is not much government promotion for this issue." (P08: male, age 25)

In fact, P04 mentioned that she frequently receives information from social media such as Facebook and Instagram rather than mass media such as television and radio. Four participants also supported P04's statement. At the same time, P03 and P04 stated that they were not aware of any promotion through social media. This category of young adults reflected that the promotion of breastfeeding by the government is not well covered in the social media. P04 had a gasp of surprise at her experience and said,

"I haven't seen this type (breastfeeding) of advertisement, because I seldom watch TV." (P04)

Regarding the breastfeeding concern, all female participants expressed that it was necessary to increase the facilities for breastfeeding mothers in public areas and in the workplace. They would like to continue breastfeeding given that they receive better supports. Moreover, even if they wanted to know more about breastfeeding, promotion and information are insufficiently provided and propagated through social and mass media in Hong Kong. Relative effects may also be factors to enhance a positive attitude to breastfeeding in public.

\subsubsection{Education and advocacy}

The remaining factor was the level of education attained by the participants. The knowledge that a participant attained through education affected how s/he would perceive, comprehend and understand breastfeeding. This, in turn, would affect how s/he reacts to breastfeeding her/ his baby. The data revealed that all participants had graduated from local mainstream schools. They mentioned that in-depth knowledge of breastfeeding was not covered in previous education curriculum in Hong Kong. In addition, teachers seldom discussed breastfeeding and sex. Some of them described: "Knowledge of breastfeeding was not included in subjects studied before, such as Biology and Liberal Studies ... limited breastfeeding information were only discussed in certain sharing session." (P04)

"The Chinese are not open minded ... breastfeeding education in Hong Kong is unclear and insufficient ... in terms of education curriculum, there is only light touch on this topic. Details are not given to students." (P13: male, age 21)
"Sexual education in Hong Kong does not cover breastfeeding either, and teachers do not talk about this in detail." (P15)

Other young adults also commented that breastfeeding should be taught in sexual education in the early stage of school studies:

"It is suggested that breastfeeding education should be included in primary education syllabus." (P15)

"Breastfeeding education or even sexual education should be educated in early childhood." (P17, female, age 25)

In addition, the parents are role models of children, so their behaviors imperceptibly affect the children in the future:

"Education given by mother is more important than education provided by schools." (P18: male, age 25)

Most of the participants emphasized that it could be a useful tool to know and obtain an in-depth understanding of breastfeeding and sex. Knowledge of breastfeeding provided for children and adolescents in Hong Kong through school life is insufficient. Providing more information on breastfeeding in mainstream schools is a needed area for improvement, as content of education curriculum only focuses on basic knowledge about sexual organs. Besides, family education is significant and important for children to build up values toward breastfeeding.

\section{Discussion}

As expected from previous quantitative studies, this article found that most Hong Kong young adults support breastfeeding. Recent statistics from the government indicated that the breastfeeding rate at 6 months of age in Hong Kong has increased from $32.7 \%$ in 2012 to $47 \%$ in $2016 .{ }^{61}$ The rise will certainly continue if there is support from the community to breastfeeding, accepting and welcoming mothers to conduct breastfeeding in public. The results of this article showed that the young men and women in Hong Kong have conflicting ideas on whether breastfeeding could be done in public. Unlike a study from the Mainland of China, ${ }^{62}$ which indicated that more than half of the participants expressed breastfeeding in public as unacceptable and indecent, the majority of young adults in Hong Kong showed support for mothers to breastfeed in public areas. However, they have reservation on that. Most of them supported breastfeeding in public, but with discretion. The discretion they 
exhibited in this article stressed secrecy or privacy (i.e. whether the mother conducts breastfeeding in private in a breastfeeding room, restroom or use special aids to cover themselves while breastfeeding in public). This situation is similar to the result of the Malaysian university students' survey, with nearly $78 \%$ of them preferring breastfeeding in public to be done in private, with discretion. ${ }^{63}$ Young adults in Hong Kong, especially the male adults, would like their spouse to breastfeed in public with privacy. Some male participants seemed to make contradictory statements when discussing their feelings and perception. The lack of consistency between supporting the ideas of women breastfeeding in public and individual's level of comfort with women breastfeeding near them is also found in similar studies. ${ }^{63,64}$ Only a few female participants showed full acceptance toward breastfeeding in public, but were concerned with the perception of the public toward it. Cultural beliefs seem to be the main reason for the inconsistency in supporting the idea, but in reality, there were contrary opinions among the male and female participants.

In Chinese culture, it is taboo to expose a woman's breasts, as they are taken as sexual provocation, and thus, breastfeeding should be hidden away from public view. Not only the Chinese, but also western countries shared similar cultural beliefs. A previous study in the United States showed that more than one-third of Americans expressed that breastfeeding should not be done in public places. ${ }^{64}$ Another recent study also showed that half of the respondents were not fully supportive of breastfeeding in public. ${ }^{55} \mathrm{~A}$ similar result was obtained in a survey among nursing undergraduate students. It was found that $41.3 \%$ of the students objected to breastfeeding in public. ${ }^{65}$ Spurles and Babineau further noted that more than two-thirds of the students thought breastfeeding should be done either in a bathroom or in a private place ${ }^{66}$ Both previous and present studies showed that most people only accepted breastfeeding in public on the condition that mothers did it with discretion.

Sustained breastfeeding for up to 6 months can be successful only if mothers can conduct breastfeeding comfortably in public places. Although most of our respondents showed supportive attitudes with breastfeeding in public, they would like the communities to take positive steps to support breastfeeding in public, like providing a lactation room or enacting relevant laws for women's right to support breastfeeding in public. The inconvenience of breastfeeding in public or even in the workplace includes the lack of a small nursery room and limited time and is a major factor for women to give up breastfeeding. To develop communities that are favorable to breastfeeding, it means dealing with the needs and concerns of different parties in the society; such as the management of shopping malls, restaurants and other public places, providing guidelines for implementing breastfeeding-friendly measures, and training staff to welcome and help breastfeeding women.

One prominent theme in the focus group interview was the criticism of the education syllabus in Hong Kong. Most participants in the interview stated that knowledge about breastfeeding in Hong Kong was under-serviced. The critics were supported by the previous study from Family Health Services in Hong Kong, ${ }^{67}$ which stated that awareness on the exclusivity and duration of breastfeeding as recommended by the World Health Organization was generally poor, particularly among young adults. Public education on the optimal infant and young child feeding practices needs to be strengthened and should be started in school. Teaching in schools improves knowledge and attitudes toward breastfeeding, and teens who hold positive attitudes to breastfeeding will more likely want their own future babies to be breastfed. Different literature reviews from past decades revealed that interventions with children ${ }^{68}$ and adolescents ${ }^{69}$ in the school environment had the potential to positively affect their breastfeeding attitudes and knowledge. Both primary and secondary school teachers were willing to incorporate infant feeding education into the classroom, and many recognized the basic benefits of breastfeeding. The idea of introducing breastfeeding into the school curriculum was under debate in other countries, as well. Further research into the attitudes, knowledge, and experiences of educators regarding teaching breastfeeding in schools will be required to increase our understanding of how best to implement lessons and curriculum in the future, and hence, improve public acceptance on breastfeeding.

\section{Implications of the study}

The findings can be used to inform and improve frontline health policy makers', family members', and health educators' understanding of Chinese young adults' perceptions about breastfeeding in public in Hong Kong. The identified factors could help young adults to appreciate a spouse's support to the mother in encouraging them to breastfeed their own babies. Young adults could also be informed that such a phenomenon is common in overseas countries. Providing and utilizing media promotion is encouraged to enable and enhance young adult's willingness to accept breastfeeding in public. The findings of the study advocated the desire among the young adults to have resources allocated and the environment adjusted in public places like offices and shopping malls to provide comfortable facilities for conducting breastfeeding in concealed places rather than in open spaces. This is especially true in the Chinese community and in Hong Kong. The findings also presented 
a need for early introduction of sex education and breastfeeding to the young generation when they are in school. This is for the benefit of nurturing the children and adolescents toward the correct understanding of sex, making a distinction between sex and breastfeeding, and hence, affecting the perception of breastfeeding positively to change the traditional cultural mindset of denying a mother breastfeeding privileges in public.

\section{Conclusions}

This focus group study explored new phenomena, such as a negative linkage between breasts and sex. There is room for improvement from the government to provide further and better supporting facilities for breastfeeding mothers, increase promotion activities related to breastfeeding, and encourage working mothers to continue breastfeeding in the workplace environment. Though young adults support breastfeeding in public, the female young adults are still concerned about exposing their breasts, as they consider it embarrassing and a taboo in the local community. To pursue in-depth and comprehensive knowledge of breastfeeding, early introduction of sex education and breastfeeding should be provided to the children and adolescents in their early stages of development. There could be a government policy to support the promotion and encouragement of breastfeeding in the community in Hong Kong. The findings in the study provided an understanding about the underlying factors that will affect young adults in accepting

\section{References}

1. DieterichCM, FeliceJP, O'SullivanE, RasmussenKM Breastfeeding and health outcomes for the mother-infant dyad. Pediatric Clin N Am. 2013;60: 31-48.

2. Victora CG, Bahl R, Barros AJ, et al. Breastfeeding in the 21st century: epidemiology, mechanisms, and lifelong effect. Lancet. 2016;387:475-490.

3. Bartick MC, Schwarz EB, Green BD, et al. Suboptimal breastfeeding in the United States: maternal and pediatric health outcomes and costs. Matern Child Nutr. 2016;13:e12366.

4. Wambach K, Riordan J. Breastfeeding and Human Lactation. Burlington, MA: Jones \& Bartlett Learning; 2016.

5. Cattaneo A, Burmaz T, Arendt M, et al. Protection, promotion and support of breast-feeding in Europe: progress from 2002 to 2007. Public Health Nutr. 2010;13:751-759.

6. Hauck Y, Fenwick L, Dhaliwal J, Butt S. A western Australian survey of breastfeeding initiation, breastfeeding in public. Through better understanding and implementation of improvement measures, it is hoped that Hong Kong could become a breastfeedingfriendly city.

\section{Author contributions}

All authors have agreed on the final version and meet at least one of the following criteria recommended by the ICMJE (http://www.icmje.org/recommendations/):

- Substantial contributions to conception and design, acquisition of data, or analysis and interpretation of data;

- Drafting the article or revising it critically for important intellectual content.

\section{Ethical approval}

The study protocol was approved by the Research and Ethics Committee, Caritas Institute of Higher Education. Potential participants received information about the study and they were informed that their response will be considered an informed consent to participate in the survey. Consent for interviews was also obtained.

\section{Conflicts of interest}

There are no conflicts of interest for any authors in this article. prevalence and early cessation patterns. Matern Child Health J. 2011;15:260-268.

7. Watt S, Sword W, Sheehan D, et al. The effect of delivery method on breastfeeding initiation from the Ontario Mother and Infant Study (TOMIS) III. J Obstet Gynecol Neonatal Nurs. 2012;41:728-737.

8. Wen LM, Simpson JM, Rissel C, Baur LA. Awareness of breastfeeding recommendations and duration of breastfeeding: findings from the Healthy Beginnings Trial. Breastfeed Med. 2012;7:223-229.

9. Baby Friendly Hospital Initiative Hong Kong Association. World Breastfeeding Week 2013: Annual Summary. Hong Kong: Baby Friendly Hospital Initiative Hong Kong Association; 2013.

10. Khin PP, Cheung SL, Loh T. Support and promotion of breastfeeding. Where are we now? Public Health Epidemiol Bull. 2002;11:25-32.

11. Lok K, Bai, D, Tarrant M. Predictors of breastfeeding initiation in Hong Kong and Mainland China born mothers. BMC Pregnancy Childbirth. 2015;15:1-11. 
12. Tarrant M, Fong DYT, Wu KM, et al. Breastfeeding and weaning practices among Hong Kong mothers: a prospective study. BMC Pregnancy Childbirth. 2010;10:1-12.

13. Chan S, Nelson E, Leung S, Li C. Breastfeeding failure in a longitudinal post-partum maternal nutrition study in Hong Kong. J Paediatr Child Health. 2000;36:466-471.

14. Dodgson J, Tarrant M, Fong D, Peng X, Hui W. Breastfeeding patterns of primiparous mothers in Hong Kong. Birth. 2003;30:195-202.

15. Tarrant M, Lok KYW, Fong DYT, et al. Effect of a hospital policy of not accepting free infant formula on in-hospital formula supplementation rates and breast-feeding duration. Public Health Nutr. 2015;18:2689-2699.

16. American Academy of Pediatrics. Policy statement: breastfeeding and the use of human milk. Pediatrics. 2012;129:e827-e841.

17. United Nations Children's Fund. Tracking Progress on Child and Maternal Malnutrition: A Survival and Development Priority; 2009. https://www.unicef.org/ publications/files/Tracking_Progress_on_Child_ and_Maternal_Nutrition_EN_110309.pdf. Accessed July 4, 2019.

18. World Health Organization. Global Strategy for Infant and Young Child Feeding. A54/INF.DOC/4. Geneva: World Health Organization; 2003.

19. Department of Health, Government of the Hong Kong Special Administrative Region. Public Perception Survey on Breastfeeding; 2015. https://www. fhs.gov.hk/english/archive/files/reports/public_perception_survey_on_breastfeeding(eng)_final.pdf. Accessed July 1, 2019.

20. Wang W, Lau Y, Chow A, Chan K. Breast-feeding intention, initiation and duration among Hong Kong Chinese women: a prospective longitudinal study. Midwifery. 2014;30:678-687.

21. Acker M. Breast is best... but not everywhere: ambivalent sexism and attitudes toward private and public breastfeeding. Sex Roles. 2009;61: 476-490.

22. Boyer K. The way to break the taboo is to do the taboo thing: breastfeeding in public and citizenactivism in the UK. Health Place. 2011;17:430-437.

23. Chen W. Understanding the cultural context of Chinese mothers' perceptions of breastfeeding and infant health in Canada. J Clin Nurs. 2010;19:1021-1029.

24. Coomson J, Aryeetey R. Perception and practice of breastfeeding in public in an urban community in Accra, Ghana. Int Breastfeed J. 2018;13:1-10.

25. Meng X, Daly A, Pollard C, Binns C, Geddes D. Community attitudes toward breastfeeding in public places among Western Australia adults, 1995-2009. J Hum Lact. 2013;29:183-189.

26. Nesbitt S, Campbell K, Jack S, Robinson H, Piehl K, Bogdan J. Canadian adolescent mothers' perceptions of influences on breastfeeding decisions: a qualitative descriptive study. BMC Pregnancy Childbirth. 2012;12:1-14.

27. Russell K, Ali A. Public attitudes toward breastfeeding in public places in Ottawa, Canada. $\mathrm{J}$ Hum Lact. 2017;33:401-408.

28. Spurles $P$, Babineau J. A qualitative study of attitudes toward public breastfeeding among young Canadian men and women. J Hum Lact. 2011;27:131-137.

29. Zhou Q, Younger KM, Kearney JM. An exploration of the knowledge and attitudes towards breastfeeding among a sample of Chinese mothers in Ireland. BMC Public Health. 2010;10:1-11.

30. Inoue M, Binns CW, OtsukaK, Jimba M, Matsubara M. Infant feeding practices and breastfeeding duration in Japan: a review. Int Breastfeed J. 2012;7:1-15.

31. Meedya S, Fahy K, Kable A. Factors that positively influence breastfeeding duration to 6 months: a literature review. Women Birth. 2010;23:135-145.

32. Thompson RE, Kildea SV, Barclay LM, Kruske S. An account of significant events influencing Australian breastfeeding practice over the last 40 years. Women Birth. 2011;24:97-104.

33. Huang P, Ren J, Liu Y, Luo B, Zhao X. Factors affecting breastfeeding adherence among Chinese mothers: a multicenter study. Medicine. 2017;96:E7619.

34. Lau Y. Breastfeeding intention among pregnant Hong Kong Chinese women. Matern Child Health J. 2010;14:790-798.

35. Lu H, Li H, Ma S, Xia L, Christensson K. Perceived family perceptions of breastfeeding and Chinese new mothers' breastfeeding behaviors. Sex Reprod Healthc. 2011;2:143-147.

36. Cohen SS, Alexander DD, Krebs NF, et al. Factors associated with breastfeeding initiation and continuation: a meta-analysis. J Pediatr. 2018;203:190-196.

37. Ku C., Chow S. Factors influencing the practice of exclusive breastfeeding among Hong Kong Chinese women: a questionnaire survey. J Clin Nurs. 2010;19:2434-2445.

38. Tarkka M. Predictors of maternal competence by first-time mothers when the child is 8 months old. J Adv Nurs. 2003;41:233-240.

39. Greene J, Stewart-Knox B, Wright M. Feeding preferences and attitudes to breastfeeding and its promotion among teenagers in Northern Ireland. J Hum Lact. 2003;19:57-65.

40. Sheehan A, Schmied V, Barclay L. Exploring the process of women's infant feeding decisions 
in the early postbirth period. Qual Health Res. 2013;23:989-998.

41. Fishbein M, Ajzen I. Belief, Attitude, Intention and Behavior: An Introduction to Theory and Research. Reading, MA: Addison-Wesley; 1975.

42. Fishbein M, Ajzen I. Predicting and Changing Behavior: The Reasoned Action Approach. New York, NY: Psychology Press; 2010.

43. Lam WWT, Fielding R, McDowell I, et al. Perspectives on family health, happiness and harmony $(3 \mathrm{H})$ among Hong Kong Chinese people: a qualitative study. Health Edu Res. 2010;27:767-779.

44. Census and Statistics Department, Government of the Hong Kong Special Administrative Region. Hong Kong Annual Digest of Statistics: 2018 Edition. Hong Kong: Statistical Information Services Section of Census Statistics Department; 2018.

45. Chuang $\mathrm{C}-\mathrm{H}$, Chang P-J, Chen Y-C, et al. Maternal return to work and breastfeeding: a population-based cohort study. Int J Nurs Stud. 2010;47:461-474.

46. Ho Y, McGrath J. Predicting breastfeeding duration related to maternal attitudes in a Taiwanese sample. J Perinat Educ. 2011;20:188-199.

47. Lööf-Johanson M, Foldevi M, Rudebeck C. Breastfeeding as a specific value in women's lives: the experiences and decisions of breastfeeding women. Breastfeed Med. 2013;8:38-44.

48. Wood NK, Woods NF, Blackburn ST, Sanders EA. Interventions that enhance breastfeeding initiation, duration, and exclusivity: a systematic review. MCN, Am J Matern Child Nurs. 2016;41: 299-307.

49. Sze KY, Chan ZCY, Chiang VCL. Women's experiences of formula feeding their infants: an interpretative phenomenological study. Front Nurs. 2018;5:49-59.

50. Walker, M. Breastfeeding management for the clinician: Using the evidence. Burlington, MA: Jones \& Bartlett Learning; 2014.

51. Lau C, Lok Y, Tarrant K. Breastfeeding duration and the theory of planned behavior and breastfeeding self-efficacy framework: a systematic review of observational studies. Matern Child Health J. 2018;22:327-342.

52. Coomson JB, Aryeetey R. Perception and practice of breastfeeding in public in an urban community, in Accra, Ghana. Int Breastfeed J. 2018;13:1-10.

53. Daly A, Pollard CM, Phillips M, Binns CW. Benefits, barriers and enablers of breastfeeding: factor analysis of population perceptions in Western Australia. PLoS ONE. 2014;9:e88204.

54. Meng X, Daly A, Pollard CM, Binns CW. Community attitudes toward breastfeeding in public places among Western Australia adults, 1995-2009. J Hum Lact. 2013;29:183-189.

55. Mulready-Ward C, Hackett M. Perception and attitudes: breastfeeding in public in New York City. J Hum Lact. 2014;30:195-200.

56. Nesbitt SA, Campbell KA, Jack SM, Robinson H, Piehl K, Bogdan JC. Canadian adolescent mothers' perceptions of influences on breastfeeding decision: a qualitative descriptive study. BMC Pregnancy Childbirth. 2012;12:149.

57. Russell K, Ali A. Public attitudes toward breastfeeding in public places in Ottawa, Canada. $J$ Hum Lact. 2017;33:401-408.

58. Boyatzis R. Transforming Qualitative Information: Thematic Analysis and Code Development. Thousand Oaks, CA: Sage; 1998.

59. QSR NVivo. Version 12.0. Melbourne, Australia: QSR International Pty Ltd; 2018.

60. Richards L. Handling Qualitative Data: A Practical Guide. Thousand Oaks, CA: Sage; 2014.

61. Family of Health Service. Breastfeeding survey 2017. Hong Kong: Department of Health; 2017. https://www.fhs.gov.hk/english/reports/files/BF_survey_2017.pdf. Accessed July 4, 2019.

62. Zhao Y, Ouyang YQ, Redding SR. Attitudes of Chinese adults to breastfeeding in public: a web-based survey. Breastfeed Med. 2017;12:316-321.

63. Aloysius M, Jamaludin SSS. Breastfeeding in public: A study of attitudes and perception among Malay undergraduates in Universiti Sains Malaysia. Geografia Malays J Soc. 2018;14:212-224.

64. Li RR, Rock VJ, Grummer SL. Changes in public attitudes toward breastfeeding in the United States, 1999-2003. J Am Diet Assoc. 2007;107:122-127.

65. Spear HJ. Baccalaureate nursing students' breastfeeding knowledge: a descriptive survey. Nurse Educ Today. 2006;26:332-337.

66. Spurles PK, Babineau J. A qualitative study of attitudes toward public breastfeeding among young Canadian men and women. J Hum Lact. 2011;27:131-137.

67. Family of Health Service. Survey on Mothers Breastfeeding Experience in Public Places. Hong Kong: Department of Health; 2016. https://www. fhs.gov.hk/english/archive/files/reports/Survey_on_ Mothers_views _on_BF_exp_in_pubplace_2016_ final.pdf. Accessed July 4, 2019.

68. Costa MM, Diniz-Santos DR, Santana JS, Silva LJ. The impact of an educational intervention on breastfeeding. Health Educ. 2006;106:309-314.

69. Zeller CL. Effects of education on breastfeeding knowledge and attitudes among middle school students. Health Educ J. 2016;75:501-510. 